\title{
VARIACIÓN ANUAL DE LA COMPOSICIÓN QUÍMICA DEL ACEITE ESENCIAL DE LIPPIA ALBA (VERBENACEAE) CULTIVADA EN COSTA RICA
}

\author{
José F. Cicció ${ }^{1} \&$ RAfael A. OCAMPO ${ }^{2}$ \\ ${ }^{1}$ Centro de Investigaciones en Productos Naturales (CIPRONA), Escuela de Química, Universidad de \\ Costa Rica, 2060 San José, Costa Rica - jfciccio@equi.ucr.ac.cr \\ ${ }^{2}$ Bougainvillea S.A., San José, Costa Rica.
}

\begin{abstract}
The variation of the annual chemical composition of the essential oil from leaves of Lippia alba (Verbenaceae) sweet form cultivated in the tropical Caribbean region of Costa Rica was determined by capillary GC/FID and GC/MS. The major constituents are carvone (62.44 - $67.55 \%)$ and limonene (20.77 $-24.95 \%)$.

Resumen. Se estudió la variación anual de la composición química del aceite esencial de las hojas de Lippia alba (Verbenaceae), forma dulce, que crece en la región tropical húmeda de Costa Rica, utilizando cromatografía de gases capilar (CG) y espectrometría de masas (EM). Los constituyentes mayoritarios del aceite son carvona $(62,44-67,55 \%)$ y limoneno $(20,77-24,95 \%)$.
\end{abstract}

Palabras Clave / Key Words: Lippia alba, Verbenaceae, aceite esencial / essential oil, cromatografía de gases / GC-MS analysis, carvona / carvone, limoneno / limonene.

\section{INTRODUCCIÓN}

La juanilama o Lippia alba (Mill.) N.E. Brown es una planta aromática que se cultiva en los jardines de muchas casas de Costa Rica, tanto en el Valle Central como en zonas del Pacífico sur y del Atlántico. Como razones para ese cultivo encontramos que la planta posee propiedades medicinales y que es apreciada por los apicultores puesto que produce gran cantidad de néctar.

En general, el uso tradicional y popular de L. alba en América está referido principalmente a la infusión de las hojas y las flores. Se emplea para trastornos gastrointestinales o digestivos, como antiespasmódico en cólicos hepáticos y como expectorante, febrífugo y sudorífico. Además, se le atribuyen actividades antisépticas, astringentes y emenagogas (Morton 1981, Gupta 1995). En Costa Rica, los usos son similares, con la adición de que se usa frecuentemente para desinflamar, contra dolores reumáticos y contra resfriados (Núñez-Meléndez 1975, Ocampo \& Maffioli 1987).

La planta pertenece a la familia Verbenaceae, en la que se incluyen cerca de 70 géneros y 2000 especies que se distribuyen en casi todas las regiones del planeta. Esta familia es más abundante en las regiones tropicales y subtropicales, donde se encuentra como hierbas, arbustos y árboles y tiene valor económico por algunos productos ornamentales y medicinales (Montiel 1991, Atkins 2004). El género Lippia está

constituido por cerca de 200 especies, que incluyen hierbas, arbustos y pequeños árboles. Estas especies se encuentran distribuidas principalmente en América Central, América del Sur y África tropical (Verdcourt 1992).

Lippia alba es un arbusto aromático, ramificado desde la base, perenne (a veces subarbusto o hierba), erecto, densamente pubescente, de hasta $2 \mathrm{~m}$ de alto, con ramas descendentes, con hojas decusado-opuestas de márgenes serrados e inflorescencias axilares, generalmente solitarias. Las flores son de color púrpura, lila o blancas. En Costa Rica se pueden identificar dos formas principales: una con un olor fragante (forma dulce) y otra con un olor sumamente fuerte y poco agradable (forma fuerte) (Ocampo \& Valverde 2000, Cicció \& Ocampo 2004). La forma fuerte está presente en el trópico seco de Costa Rica (1200 mm de precipitación), con ramas péndulas, raíces adventicias en los nudos, hojas de color verde oscuro, largas y angostas, con entrenudos largos y con un aroma fuerte y áspero. La forma dulce se encuentra en el trópico húmedo (3000-4000 $\mathrm{mm}$ de precipitación), con ramas erectas, entrenudos cortos, hojas de color verde claro, redondas, con un aroma suave y dulce. Se considera que esta forma fue introducida de Panamá. En la literatura se encuentran más de 15 sinónimos para esta especie, que ha sido ubicada anteriormente en los géneros Lantana, Verbena y Zapania (Gupta 1995). Se 
encuentra desde regiones secas en América del Norte, sur de Estados Unidos (Texas) y México, América Central, hasta Argentina, y en regiones húmedas como la Amazonia y las islas del Caribe.

\section{Composición QuímICA DE LOS ACEITES ESENCIALES}

Los aceites esenciales de esta planta se caracterizan por poseer una composición muy variable, dependiendo del lugar de procedencia (Velasco-Negueruela et al. 1993, Terblanché \& Cornelius 1996). Por ejemplo, en plantas que crecen en Argentina se encontraron varios quimiotipos (que son plantas de una misma especie que se diferencian entre sí porque presentan una distinta composición química del aceite), que tienen como constituyentes mayoritarios lippiona (1,2-epoxipulegona) (Retamar 1994), dihidrocarvona (Fester et al. 1961a), citral y 1,8-cineol (Fester et al. 1961b), y un cuarto tipo con piperitona (36,7\%) y limoneno (34,2\%) (Catalán et al. 1977).

En la región de São Paulo, al sureste de Brasil (Frighetto et al. 1998, Siani et al. 2002) se encontró otro tipo, que contiene como constituyentes principales $S$-linalol (78,9\%) y 1,8-cineol $(6,5 \%)$, hallado también en plantas obtenidas en condiciones experimentales, provenientes de Paraje Las Brujas, Canelones, Uruguay (Lorenzo et al. 2001) con más del 55\% de linalol. Ricciardi et al. (1999) determinaron tres quimiotipos de Lippia alba en el Chaco y en Corrientes (región del nordeste de Argentina), sitio de origen de las plantas. Se demostró la existencia del quimiotipo citral en las plantas del Chaco, correlacionado con el que Fester llamó quimiotipo del norte de Santa Fe y Entre Ríos (Fester et al. 1961). La determinación de la distribución enantiomérica del linalol en $L$. alba del Chaco, en verano, permitió corroborar que se trata principalmente de $3 S-(+)$-linalol, que coincide con lo observado por Siani et al. (2002) en la misma especie procedente de Brasil y por Lorenzo et al. (2001) en especies silvestres de Uruguay. Se informó de un quimiotipo de plantas que crecen en Uruguay, en el que los constituyentes principales del aceite son alcanfor (18,2\%) y 1,8-cineol (16,5\%) (Dellacassa et al. 1990), además de la existencia del quimiotipo linalol (Lorenzo et al. 2001).El aceite de L. alba recolectada en Manaus, Brasil (Craveiro et al. 1981), posee $\beta$-cariofileno $(24,3 \%)$, geranial $(12,9 \%)$, neral $(9,6 \%)$ y 2 -undecanona $(9,0 \%)$, mientras que otro tipo químico de Brasil, cultivado en Paraná, presentó $\gamma$-terpineno $(46,7 \%)$, $p$-cimeno $(8,7 \%)$ y $\beta$-cariofileno $(7,2 \%)$ como constituyentes mayoritarios (Gómes et al. 1993). Las plantas cultivadas en la región noreste de Brasil presentaron dos quimiotipos: uno con neral
$(27,2-30,4 \%)$, geranial $(35,6-41,0 \%)$ y limoneno $(9,1-$ $13,1 \%)$ y otro con carvona $(42,3-54,7 \%)$, limoneno $(23,2-30,4 \%)$ y $p$-cimeno $(3,1-10,6 \%)$ (Fun \& Baerheim-Svendsen 1990, Matos et al. 1996).

En Cuba (Pino \& Ortega 1996, Pino et al. 1996) se informó de un tipo semejante a este último de Brasil, que presentó carvona $(29,0-40,0 \%)$, piperitenona $(6,4-8,3 \%)$, limoneno $(5,8-6,5 \%)$ y $\beta$-guaieno $(9,8$ $11,5 \%$ ) como compuestos mayoritarios. El quimiotipo carvona también se encontró en plantas recolectadas en Iquitos, Perú (Leclercq et al. 1999) con un 63,4\% de carvona, 5,6\% de germacreno D y un 5,1\% de limoneno. También en material cultivado en la región caribe de Costa Rica se encontró el quimiotipo carvona-limoneno (Cicció \& Ocampo 2004), que también se encontró en Bucaramanga, Colombia, donde se determinó que el aceite es rico en $S$-carvona (41 - 57\%) (Stashenko et al. 2004).

En América del Norte, el aceite esencial obtenido de plantas cultivadas en macetas (en Delaware), procedentes de Sinaloa, México, dio un nuevo quimiotipo en el que los constituyentes mayoritarios son metilchavicol (estragol) (56,5\%), 1,8-cineol (12,6\%) y canfeno (7,0\%) (Tucker \& Maciarello 1999). En Guatemala se encontró un quimiotipo con neral $(17,6$ - $18,9 \%)$, geranial $(24,7-27,0 \%)$ y 1,8 -cineol $(20,3$ $25,4 \%$ ) (Fischer et al. 2004). Un nuevo quimiotipo que contiene mircenona $(37,8-58,2 \%)$ y $(Z)$-ocimenona $(11,1-16,3 \%)$ se encontró en Guatemala (Fischer et al. 2004), en las regiones del Pacífico y del centro de Costa Rica (Cicció \& Ocampo 1998) y en la provincia de Corrientes, Argentina (Richiardi et al. 1999).

\section{IMPORTANCIA ECONÓMICA}

La planta tiene importancia económica en América Central, ya que se vende en los mercados populares y se emplea en la industria de tisanas (CATIE 1995) y en preparaciones medicinales. En Colombia, la industria emplea material para tisanas con propósito digestivo, para comprimidos y encapsulados así como en sales para baños y para cremas (Gutiérrez \& Duque 2003). En el mercado de Costa Rica, se encuentra en la forma comercial de bolsitas-filtro, para infusión y como parte de la fórmula de algunos productos para el tratamiento de resfriados (congestión de vías respiratorias) y contra el reumatismo (preparaciones con base en extractos alcohólicos, algunas veces combinados con extractos de saragundí (Senna alata, Fabaceae) y de chile (Capsicum spp., Solanaceae). En la Farmacopea Vegetal Caribeña se indica la decocción de la hoja fresca, vía oral, como un uso tradicional significativo para la gripe y el resfriado (Germosén-Robineau 2005). 
Recientemente ha aparecido un resumen de mercado basado en el quimiotipo carvona (UNCTAD 2005).

Este trabajo tiene como propósito el estudio de la variación en la composición de los aceites esenciales obtenidos del material proveniente de cinco cosechas efectuadas en el transcurso de un año, a partir de una plantación en la zona atlántica de Costa Rica, que corresponde al quimiotipo carvona-limoneno (forma dulce de Lippia alba). Los resultados son relevantes desde el punto de vista de la calidad del material de cultivo (domesticación), ya que permite determinar si, durante el año, en la materia prima ocurren cambios importantes en la composición química de los aceites.

\section{PARTE EXPERIMENTAL}

Material vegetal: El material vegetal provino de parcelas cultivadas por Bougainvillea S.A., Costa Rica, de plantas originalmente procedentes de la región de Talamanca, provincia de Limón (zona sureste de Costa Rica). El cultivo está establecido en la región tropical húmeda caribeña, con una altitud de $100 \mathrm{~m}$, una temperatura media de $22{ }^{\circ} \mathrm{C}$ y una precipitación media de $3000 \mathrm{~mm}$ (localización $10^{\circ} 01^{\prime} 03.1^{\prime \prime} \mathrm{N}$, $83^{\circ} 19^{\prime} 41.6^{\prime \prime} \mathrm{W}$ ).

Las plantas se caracterizan por el crecimiento erecto, entrenudos cortos y hojas cortas con un olor fragante y dulce. Se estudiaron muestras de cultivo recogidas en junio, septiembre y diciembre de 2003, así como en febrero y abril de 2004. Todas las muestras se secaron a la sombra durante una semana. Un ejemplar testigo se depositó en el Herbario de la Universidad de Costa Rica (USJ-70741).

EXTRACCIÓN: Los aceites esenciales de las hojas secas fueron extraídos mediante el procedimiento de hidrodestilación, durante $3 \mathrm{~h}$, empleando para ello un equipo de Clevenger modificado. Los aceites, levemente amarillentos, se secaron sobre sulfato de sodio anhidro, obteniéndose rendimientos que oscilan entre 2,0 y $2,7 \%(\mathrm{v} / \mathrm{m})$.

ANÁlisis CG/FID: Los análisis de los aceites de $L$. alba se efectuaron usando un equipo Shimadzu GC17. Los datos se obtuvieron en una columna capilar de sílice fundida con un recubrimiento interno de 5\% fenil metilpolisiloxano $(30 \mathrm{~m} \times 0.25 \mathrm{~mm} \mathrm{~d}$. i., grosor de película de $0.20 \mu \mathrm{m}$ ), Heliflex (Alltech) AT-5, y con el software Shimadzu Class-VP, versión 4.3. Las condiciones de operación fueron: gas portador: $\mathrm{N}_{2}$
$(1.0 \mathrm{~mL} / \mathrm{min})$; temperatura del horno, programación: $60-220^{\circ} \mathrm{C}$ a $3^{\circ} \mathrm{C} / \mathrm{min}, 220^{\circ} \mathrm{C}$; temperatura del puerto de inyección de muestra: $250{ }^{\circ} \mathrm{C}$; temperatura del detector: $275^{\circ} \mathrm{C}$; sistema de inyección split 1: 50 .

ANÁLisis CG/EM: El análisis por CG/EM se efectuó utilizando un equipo Shimadzu GC-17A acoplado con el aparato GCMS-QP5050 y utilizando el software CLASS 5000 con la base de datos Wiley138. Los datos se obtuvieron en una columna capilar de sílice fundido ( $30 \mathrm{~m} \times 0.25 \mathrm{~mm}$; grosor de película de $0.25 \mu \mathrm{m}$ ) y con un recubrimiento interno de $5 \%$ fenil metilpolisiloxano. Las condiciones de operación fueron: gas portador $\mathrm{He}$, flujo $1.0 \mathrm{~mL} / \mathrm{min}$; temperatura del horno, programación: $60-240^{\circ} \mathrm{C}$ a $3{ }^{\circ} \mathrm{C} / \mathrm{min}$; temperatura del puerto de inyección de muestra: $250^{\circ} \mathrm{C}$; temperatura del detector: $260^{\circ} \mathrm{C}$; voltaje de ionización: $70 \mathrm{eV}$; corriente de ionización $60 \mu \mathrm{A}$; velocidad de barrido $0.5 \mathrm{~s}$ sobre un ámbito de 38-400 amu; split 1:70.

IDENTIFICACIÓN: Los constituyentes de los aceites se identificaron mediante sus índices de retención, por comparación de sus espectros de masas con los publicados en la literatura (McLafferty 1993, Adams 2001) y por comparación visual usando nuestra propia base de datos.

\section{RESULTADOS Y DISCUSIÓN}

Los datos obtenidos para los compuestos mayoritarios se muestran en el Cuadro 1. Como se observa del análisis de las muestras, la planta pertenece al quimiotipo carvona-limoneno. Por tanto, el biotipo de planta (Lippia alba, forma dulce) que se encuentra en forma natural en el sureste de Costa Rica se asemeja a los quimiotipos registrados en Colombia (Stashenko et al. 2004), Brasil (Matos et al. 1996), Perú (Leclercq et al. 1999) y Cuba (Pino \& Ortega 1996, Pino et al. 1996); sin embargo, se diferencia del de Cuba, por ejemplo, en que no presenta $\beta$-guaieno. Los dos constituyentes mayoritarios del aceite esencial se mantuvieron dentro de los siguientes límites: 62,44 $\%$ a $67,55 \%$ de carvona y $20,77 \%$ a $24,95 \%$ de limoneno.

Se puede concluir que la composición química del aceite es notablemente estable durante el año. Por tanto, en esa región específica de la zona atlántica de Costa Rica la cosecha de la planta se podría efectuar durante distintos meses del año sin que haya una gran variación en la composición del aceite. Esto parece indicar que se tiene una calidad consistente de la planta seca, lo cual 
es muy importante para la estandarización de extractos y de aceite esencial con propósitos comerciales.

La gran diversidad química de esta especie parece reflejar su capacidad de respuesta a las diversas condiciones extrínsecas del medio ecológico (coordenadas geográficas, clima, suelo, exposición solar, etc.), ya que se encuentra tanto en regiones tropicales como subtropicales del continente americano; también podría reflejar una gran plasticidad genética propia de esta especie, que probablemente le permite adaptarse con mayor facilidad a diversos hábitats del continente.

Cuadro 1. Variación porcentual de los constituyentes mayoritarios de Lippia alba (Verbenaceae) cosechada durante diversos meses en la bajura atlántica de Costa Rica $\left(10^{\circ} 01\right.$ '03.1”N, 83¹9’41.6”W).

\begin{tabular}{|l|r|r|r|r|r|}
\hline Constituyente & $\begin{array}{r}\text { junio } \\
\%\end{array}$ & $\begin{array}{r}\text { septiembre } \\
\%\end{array}$ & $\begin{array}{r}\text { diciembre } \\
\%\end{array}$ & $\begin{array}{r}\text { febrero } \\
\%\end{array}$ & $\begin{array}{r}\text { abril } \\
\%\end{array}$ \\
\hline & & & & & \\
\hline mirceno & 1,24 & 0,80 & 1,11 & 1,23 & 1,00 \\
\hline limoneno & 24,95 & 20,77 & 24,70 & 24,19 & 22,32 \\
\hline linalol & 0,56 & 0,33 & 0,39 & 0,55 & 0,51 \\
\hline carvona & 63,92 & 67,55 & 64,17 & 62,44 & 62,58 \\
\hline piperitona & 0,44 & 0,56 & 0,44 & 0,64 & 0,42 \\
\hline$\beta$-bourboneno & 0,82 & 1,48 & 1,06 & 1,33 & 1,49 \\
\hline$\beta$-cariofileno & 0,87 & 1,25 & 1,07 & 1,26 & 1,43 \\
\hline E- $\beta$-farneseno & 0,36 & 0,59 & 0,44 & 0,59 & 0,83 \\
\hline germacreno D & 2,73 & 3,05 & 2,78 & 3,66 & 4,26 \\
\hline
\end{tabular}

Agradecimientos. Agradecemos al CYTED, Proyecto IV.20 (Normalización de productos naturales obtenidos de especies de la flora aromática latinoamericana) por permitir nuestra participación en este proyecto. Agradecemos a Lorena Hernández, Grettel Morales y Carlos Chaverri por su colaboración.

\section{LITERATURA CITADA}

Adams, R.P. 2001. Identification of essential oil components by gas chromatography/ Quadrupole mass spectroscopy. Allured Publish., Carol Stream, IL.

Atkins, S. 2004. Verbenaceae. In: Smith, N., S.A. Mori, A. Henderson, D.W. Stevenson \& S.V. Heald (eds.). Flowering plants of the New Tropics. The New York Bot. Gard., Princeton Univ. Press. p. 387-390.

Catalán, C.A.N., D.J. Merep \& J.A. Retamar. 1977. Aceite esencial de Lippia alba (Miller) N.E. Brown de la provincia de Tucumán. Riv. Ital. EPPOS 59: 513-518.

CATIE. 1993. Caracterización de los sectores asociados a la producción, comercialización y transformación de plantas medicinales. Mimeografiado. 35 p.
Cicció, J.F. \& R.A. Ocampo. 1998. Estudio del aceite esencial de Lippia alba (Mill.) N.E. Br. ex Britton \& Wilson (Verbenaceae) de Costa Rica. Informe parcial Proyecto IV.6. La flora Iberoamericana y su aprovechamiento para la producción de aromas y fragancias de interés industrial (mimeografiado).

Cicció J.F. \& R.A. Ocampo. 2004. Aceite esencial de Lippia alba (Verbenaceae) cultivada en el trópico húmedo en el Caribe de Costa Rica. Ing. Cien. Quím. 21: 13-16.

Craveiro, A.A., J.W. Alencar, F.J.A. Matos, C.H.S. Andrade \& M.I.L. Machado. 1981. Essential oils from Brazilian Verbenaceae. Genus Lippia. J. Nat Prod. 44(5): 598-601.

Dellacassa, E., E. Soler, P. Menéndez \& P. Moyna. 1990. Essential oils of Lippia alba (Mill.) N.E. Brown and Aloysia chamaedrifolia Cham. (Verbenaceae) from Uruguay. Flav. Fragr. J. 5: 107-108.

Fester, G., J. Retamar, A. Ricciardi \& A. Cassano. 1961. Algunas esencias volátiles. 14 . Comunicación. Rev. Fac. Ing. Quím. 30: 5-10.

Fester, G., L.R. Fonseca, A. Ricciardi, A. Cassano \& J. Burgos. 1961. Algunas esencias volátiles. 15ª Comunicación. Rev. Fac. Ing. Quím. 30: 11-14. 
Fischer, U., R. López, E. Pöll, S. Vetter, J. Novak \& C.M. Franz. 2004. Two chemotypes within Lippia alba populations in Guatemala. Flavour Fragr. J. 19: 333-335.

Frighetto, N.J.G. de Oliveira, A.C. Siani \& K.C. das Chagas. 1998. Lippia alba (Mill.) N.E. Br. (Verbenaceae) as a source of linalool. J. Essent. Oil Res. 10: 578-580.

Fun, C.E. \& A. Baerheim Svendsen. 1990. The essential oil of Lippia alba (Mill.) N.E. Br. J. Essent. Oil. Res. 2: 265-267.

Germosén-Robineau, L. (ed.). 2005. Farmacopea Vegetal Caribeña. 2a ed. TRAMIL, Edit. Universitaria, UNAN, León, Nicaragua. p. 254257.

Gómes, E.C., L.C. Ming, O.E.A. Moreira, G. Miguel, M.D. Miguel, V.A. Kerver, A. Conti \& A.W. Filho. 1993. Constituents of the essential oil from Lippia alba (Mill.) N.E. Br. (Verbenaceae). Rev. Brasil. Farm. 74(2): 29-32.

Gupta, M.P. (ed.). 1995. 270 Plantas Medicinales Iberoamericanas. CYTED-SECAB, Santafé de Bogotá, Colombia.

Gutiérrez-Domínguez, M.A. \& A. Duque-Villegas. 2003. Estudio básico de mercado de ocho plantas medicinales de interés latinoamericano. Proyecto OEA-AICD / AE-089/02.

Leclercq, P.A., H. Silva Delgado, J. García, J.E. Hidalgo, T. Cerrutti, M. Mestanza, F. Ríos, E. Nina, L. Nonato, R. Alvarado \& R. Menéndez. 1999. Aromatic plant oils of the Peruvian Amazon. Part 1. Lippia alba (Mill.) N.E. Br. and Cornutia odorata (Poeppig) Poeppig ex Schauer, Verbenaceae. J. Essent. Oil. Res. 11: 753-756.

Lorenzo, D., D. Paz, P. Davies, R. Vila, S. Cañigueral \& E. Dellacassa. 2001. Composition of a new essential oil type of Lippia alba (Mill.) N.E. Brown from Uruguay. Flavour Fragr. J. 16: 356-359.

Matos, F.J.A., M.I.L. Machado, A.A. Craveiro \& J.W. Alencar. 1996. Essential oil composition of two chemotypes of Lippia alba grown in Northeast Brazil. J. Essent. Oil. Res. 8: 695-698.

McLafferty, F.W. 1993. Registry of Mass Spectral Data. John Wiley \& Sons, New York.

Montiel, M. 1991. Introducción a la flora de Costa Rica. Edit. UCR, San José.

Morton, J.F. 1981. Atlas of Medicinal Plants of Middle America. Bahamas to Yucatan. Charles C. Thomas, Springfield, IL.

Núñez-Meléndez, E. 1975. Plantas medicinales de Costa Rica y su folclore. Edit. UCR, San José.

Ocampo, R.A. \& A. Maffioli. 1987. El uso de algunas plantas medicinales en Costa Rica. Vol. I, $2^{\mathrm{a}}$ ed. Edit. Trejos Hnos., San José. p. 51-53.

Ocampo, R.A. \& R. Valverde. 2000. Manual de cultivo y conservación de plantas medicinales. Tramil, San José.

Pino, J.A. \& A. Ortega. Chemical Composition of the Essential Oil of Lippia alba (Mill.) N.E. Brown from Cuba. J. Essent. Oil Res. 8: 445-446.

Pino, J.A., A.G. Ortega, A. Rosado, M. Rodríguez \& R. Baluja. 1996. Composición y propiedades antibacterianas del aceite esencial de Lippia alba (Mill.) N.E. Brown. Rev. Cubana Farm. 30(1).

Retamar, J.A. 1994. Variaciones fitoquímicas de la especie Lippia alba (salvia morada) y sus aplicaciones en la química fina. Essenze Deriv. Agrum. 64: 55-60.

Ricciardi, G.A.L., J.F. Veglia, A.I.A. Ricciardi \& A.L. Bandoni. 1999. Examen comparado de la composición de los aceites esenciales de especies autóctonas de Lippia alba (Mill.) N.E. Br. Univ. Nac. Nordeste, Comunicaciones Científicas y Tecnológicas. (www.unne.edu.ar/cyt/exactas/e-029. pdf).

Siani, A.C., M.M.R. Tappin, M.F.S. Ramos, J.L. Mazzei, M.C.K.V. Ramos, F.R. de Aquino Neto \& N. Frighetto. 2002. Linalol from Lippia alba: Study of the reproducibility of the essential oil profile and the enantiomeric purity. J. Agric. Food Chem. 50: 3518-3521.

Stashenko, E.E., B.E. Jaramillo \& J.R. Martínez. 2004. Comparison of different extraction methods for the analysis of volatile secondary metabolites of Lippia alba (Mill.) N.E. Brown, grown in Colombia, and evaluation of its in vitro antioxidant activity. J. Chromatog. 1025: 93-103.

Terblanché, F.C. \& G. Kornelius. 1996. Essential oil constituents of the genus Lippia (Verbenaceae). A Literature review. J. Essent. Oil Res. 8: 471-485.

Tucker, A.O. \& M.J. Maciarello. 1999. Volatile Leaf Oil of the "Licorice Verbena" (Lippia alba (Mill.) N.E. Brown ex Britton and P. Wils. var. carterae Moldenke) from the North American Herb Trade. J Essent. Oil Res. 11: 314-316.

UNCTAD. 2005. Market Brief in the European Union for selected natural ingredients derived from native species. Lippia alba. Prontoalivio, Erva cidreira, juanilama, Melissa. The United Nations Conference on Trade and Development (UNCTAD). BioTrade Initiative / BioTrade Facilitation Program (BTFP). $39 \mathrm{p}$.

Velasco-Negueruela, A., M.J. Pérez-Alonso, C.A. Guzmán, J.A. Zigadlo, L. Ariza-Espinar, J. Sanz \& 
M.C. García-Vallejo. 1993. Volatile Constituents of Verdcourt, B. 1992. Flora of Tropical East Africa. Four Lippia Species from Córdoba (Argentina). J. Verbenaceae. A.A. Balkema, Rotterdam / BroodEssent. Oil Res. 5: 513-524. field. 